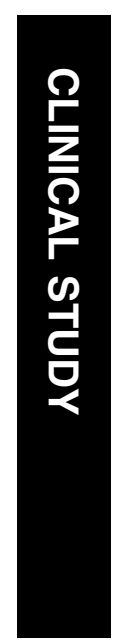

\title{
Survival analysis of 365 patients with exotropia after surgery
}

${ }^{1}$ Department of Ophthalmology, Seoul National University College of Medicine, Seoul, Republic of Korea

${ }^{2}$ Seoul Artificial Eye Center, Seoul National University Hospital Clinical Research Institute, Seoul, Republic of Korea

${ }^{3}$ Seoul National University Bundang Hospital, Seongnam, Republic of Korea

Correspondence: J-M Hwang, Department of Ophthalmology, Seoul National University College of Medicine, Seoul National University Bundang Hospital, 300, Gumi-dong, Bundang-gu, Seongnam, Gyeonggi-do 463-707,

Republic of Korea

Tel: + 8231787 7372;

Fax: + 82317874057

E-mail: hjm@snu.ac.kr

Received: 13 April 2005

Accepted in revised form:

3 August 2005

Published online:

16 September 2005

The authors have no financial conflict of interest to declare regarding the subject of this manuscript

\begin{abstract}
Purpose Few reports have included large numbers of exotropia patients. Thus, we undertook this study to perform a survival analysis of over 350 patients with exotropia and to determine which factors might affect the outcome of exotropia surgery.

Methods The clinical records of 365 patients who underwent exotropia surgery by one surgeon were retrospectively reviewed.

Preoperative patient characteristics, surgical procedures performed, and early postoperative ocular alignment were evaluated as potential risk factors of surgical outcome using survival analysis.
\end{abstract}

Results The estimated median time from surgery to recurrence was 48.3 months. None of the characteristics or procedures were found to be significantly associated with surgical outcome. The likelihood of a good postoperative surgical outcome was highest with an initial postoperative alignment of more than 10 prism diopters of esotropia $(P<0.001)$.

Conclusions Early postoperative overcorrection was the only predictor of a successful long-term outcome after exotropia surgery.

Eye (2006) 20, 1268-1272. doi:10.1038/sj.eye.6702091; published online 16 September 2005

Keywords: exotropia; outcome; surgery; survival analysis

\section{Introduction}

Results of exotropia are sometimes frustrating, principally because of the high rate of long-term undercorrection. ${ }^{1-6}$ The factors reported to affect surgical outcome after exotropia surgery vary widely in reports, ${ }^{1-10}$ and no consensus has been reached concerning predictor variables. In addition, few reports on survival analysis in a large number of patients with exotropia are available. Therefore, a survival analysis of surgical outcome and the determination of preoperative and intraoperative factors affecting surgical outcome would be helpful for planning surgical intervention and for setting realistic expectations for both patients and doctors. This study was undertaken to determine the results of exotropia surgery and to identify those factors affecting surgical outcome in over 350 patients. This study includes the largest numbers of exotropia patients ever examined in a single study.

Patients and methods

This retrospective study comprised 365 consecutive patients who underwent surgery for primary exotropia by one surgeon (JMH) between January 1998 and February 2003.

Clinical records were reviewed and various preoperative patient characteristics, details of the surgical procedures performed, and early postoperative ocular alignment were collected retrospectively as factors potentially predictive of surgical outcome.

Patients were excluded if they had a history of prior strabismus surgery, paralytic or restrictive exotropia, ocular pathology, chromosomal anomalies, or systemic disorders such as congenital anomalies or neurological disorders. Patients with A or V patterns, dissociated vertical deviation, or oblique muscle overactions were not excluded.

All patients underwent complete ophthalmologic examinations before the operation. Refractive errors were determined by cycloplegic refraction with cyclopentolate hydrochloride $1 \%$ and analysed as spherical equivalent values; myopia was represented as negative numbers and hyperopia as positive numbers. Prism and alternate cover testing 
(with accommodative targets for fixation at $1 / 3$ and $6 \mathrm{~m}$ in most patients), and a modified Krimsky method at $1 / 3 \mathrm{~m}$ in a few uncooperative patients, were conducted. An additional prism and alternate cover test was performed for cooperative patients while they looked through a window and fixated on a distant target. An additional near measurement was obtained after $1 \mathrm{~h}$ of monocular occlusion of the habitually deviating eye, and another postocclusion near measurement was obtained with an additional +3.00 diopters (D) sphere over each eye prior to allowing the patient to regain binocular fusion. Anisometropia was defined as a spherical or cylindrical difference of at least $2.00 \mathrm{D}$ between the two eyes, and amblyopia was defined as a difference of two or more lines between monocular visual acuities. Sensory status was evaluated in the cooperative patients using the Titmus stereotest and the Worth 4-Dot test at distance and near. Stereopsis was arbitrarily defined as good if there were more than $100 \mathrm{~s}$ of arc. Constant deviation was defined as being divergent for $100 \%$ of waking hours, and the constancy of deviation was assessed by noting how frequently the strabismus was observed at home and in the clinic by the parents or the ophthalmologist.

Patients with hyperopia of $>+3.00 \mathrm{D}$, myopia of $\geq-1.00 \mathrm{D}$, or astigmatism of $\geq \pm 1.50 \mathrm{D}$ or more were prescribed glasses before a final surgical decision was made. In patients with hyperopia, glasses of approximately +1.00 to $+1.50 \mathrm{D}$ less than the full cycloplegic hyperopic refraction were given. Part-time occlusion therapy was attempted in patients whose parents wanted to try a nonsurgical treatment first or to postpone surgical intervention. ${ }^{11}$ The period of part-time occlusion was 3-18 months until parents wanted to discontinue or the exotropia was found to have improved.

Surgery was recommended if there was a deterioration in the frequency or magnitude of exotropia despite nonsurgical therapy such as alternate occlusion or minus lens therapy in most patients, or if tropia was present more than $50 \%$ of the time as determined either by examination or by obtaining an ophthalmic history. All surgeries were performed under general anaesthesia by the same surgeon (JMH) using the same surgical table. ${ }^{12}$ Surgical dosage was based on distance measurements. Patients with amblyopia underwent a unilateral lateral rectus recession and a medial rectus resection. Patients with exotropia of 15-20 prism diopters (PD) underwent unilateral lateral rectus recession. Patients whose exotropia at near was greater than at distance by more than 15 PD underwent bilateral medial rectus resection. Patients with exotropia of more than 70 PD underwent bilateral lateral rectus recession with unilateral medial rectus resection. Otherwise, bilateral lateral rectus surgery was preferred to maintain the greatest range of extraocular motility. Surgery was performed for the largest angle of deviation ever measured. ${ }^{13}$

All patients were seen 1 day after the operation, at which time initial alignments at distance and near were recorded. Final alignment at distance fixation was recorded at the last examination. Postoperative measurements were taken in the same manner as the preoperative measurements. Patients with diplopia associated with postoperative esotropia were managed by alternating full time patching for 1-4 weeks until diplopia was resolved. If the esotropia did not reduce with alternate patching for 4 weeks, cycloplegic refraction was reperformed and hyperopia of $>+1.00 \mathrm{D}$ was corrected. Patients without hyperopia of $>+1.00 \mathrm{D}$ were prescribed base-out Fresnel press-on prisms (3M Health Care, St Paul, MN, USA) to allow constant fusion. When it became evident that prisms would have to be worn for a number of months, a prism incorporated into a regular spectacle was prescribed. Bifocal correction or echothiopate iodide was prescribed if a patient showed orthophoria to small esophoria at distance and a larger angle of esotropia at near.

Patients were assigned to one of the following surgical outcome groups based on the last measurement of deviations available during the postoperative visit: (1) success; (2) recurrence; (3) overcorrection. Success was defined as distant alignment in primary position, with correction if necessary, of esotropia/phoria $<6$ PD or exotropia/phoria $<11$ PD.

Recurrence was defined as a final alignment of $>10$ PD of exotropia/phoria and an overcorrection defined as $>5$ PD of esotropia/phoria. Reoperation was performed if esotropia of 20 PD or more persisted or increased for more than 6 months postoperatively. Patients who required reoperation were, by definition, all counted as recurrences before the time of the second surgery.

Survival analysis was performed to assess the surgical outcome with time, and Cox multivariate survival analysis and the generalized Wilcoxon test were used to identify factors significantly affecting outcome. The predictor variables that were chosen and used throughout the statistical analysis included age at deviation onset, age at surgery, interval between deviation onset and surgery, preoperative deviation at distance and near, refractive errors, presence of amblyopia or anisometropia, type of exotropia according to the Burian classification, ${ }^{14}$ constancy of deviation (intermittent or constant), presence of lateral incomitance, preoperative stereopsis status, presence of associated strabismus such as dissociated vertical 
deviation, $\mathrm{A}$ or $\mathrm{V}$ pattern, inferior oblique overaction, and vertical deviation.

\section{Results}

Patient demographics and characteristics are shown in Table 1. In all, 220 patients (60.3\%) had a successful outcome, 129 patients (35.3\%) had recurrence, and 16 patients $(4.4 \%)$ overcorrection. The average postoperative follow-up was 18.4 months (range 0.5 to 99 months). Survival analysis showed that the recurrence rates increased with time and that median recurrence time from surgery to recurrence of deviation was 48.3 months (Figure 1).

\section{Preoperative factors}

Of the preoperative variables investigated, none was found to be significantly associated with surgical outcome by Cox multivariate survival analysis.

\section{Surgical procedures used}

The procedure chosen did not influence success. In all, 264 patients were treated with bilateral lateral rectus recessions and 58 patients with recess/resect procedures. Unilateral lateral rectus recession was performed in 26 patients, bilateral medial rectus resection in nine, and bilateral lateral rectus recession with unilateral medial rectus resection in eight. Of the bilateral lateral rectus recessions performed, 19 cases were combined with a vertical transposition of horizontal rectus and 52 cases were combined with surgery of the vertical rectus or oblique muscle. When results of bilateral lateral rectus recession alone and in combination with vertical transposition of the horizontal rectus or surgery of the vertical rectus or oblique muscle were compared, no statistically significant difference was found in terms of surgical outcome.

\section{Early postoperative alignment}

Deviations at postoperative day 1 are shown in Table 2. The chance of a successful outcome was highest in patients with an initial alignment of $>10 \mathrm{PD}$ of esotropia/phoria $(P<0.001)$.

The median recurrence time was 99.0 months in patients with an initial alignment of $>10 \mathrm{PD}$ of esotropia/phoria, 34.1 months in those with $0-10 \mathrm{PD}$ of esotropia/phoria, and 13.2 months in those with 1-10 PD of exotropia/phoria (Figure 2). Compared to patients with an initial alignment of more than 10 PD of exotropia/phoria, the odds of a successful outcome were 7.2-fold higher in patients with an initial alignment of
Table 1 Demographics and preoperative characteristics of the 365 patients

\begin{tabular}{|c|c|}
\hline $\begin{array}{l}\text { Demographics } \\
\text { Age at surgery (years) } \\
\text { mean } \pm \text { SD (range) }\end{array}$ & $9.0 \pm 9.1(1-61)$ \\
\hline $\begin{array}{l}\text { Age at onset (years) } \\
\text { mean } \pm \text { SD (range) }\end{array}$ & $4.3 \pm 4.9(0-32)$ \\
\hline Sex male: female & $184: 181$ \\
\hline Ocular characteristics (no. of & \\
\hline $\begin{array}{l}\text { Preoperative deviation at } \\
\text { distance (prism diopters) }\end{array}$ & $36.7 \pm 12.1$ \\
\hline $\begin{array}{l}\text { Preoperative deviation at near } \\
\text { (prism diopters) }\end{array}$ & $33.0 \pm 13.9(0-95)$ \\
\hline Type of exotropia & \\
\hline Basic & $290(79.5 \%)$ \\
\hline Simulated divergence excess & $35(9.6 \%)$ \\
\hline True divergence excess & $16(4.4 \%)$ \\
\hline Convergence insufficiency & $13(3.6 \%)$ \\
\hline Constant deviation & $37(10.1 \%)$ \\
\hline Amblyopia & $85(23.3 \%)$ \\
\hline Anisometropia & $20(5.5 \%)$ \\
\hline Stereopsis status & \\
\hline$\leq 100 \mathrm{~s}$ & $145(39.7 \%)$ \\
\hline$>100 \mathrm{~s}$ & $159(43.6 \%)$ \\
\hline Associated strabismus (no. of & \\
\hline Dissociated vertical deviation & $28(7.7 \%)$ \\
\hline Inferior oblique overaction & $43(11.8 \%)$ \\
\hline Vertical deviation & $14(3.8 \%)$ \\
\hline A or $\mathrm{V}$ pattern & $56(15.3 \%)$ \\
\hline
\end{tabular}

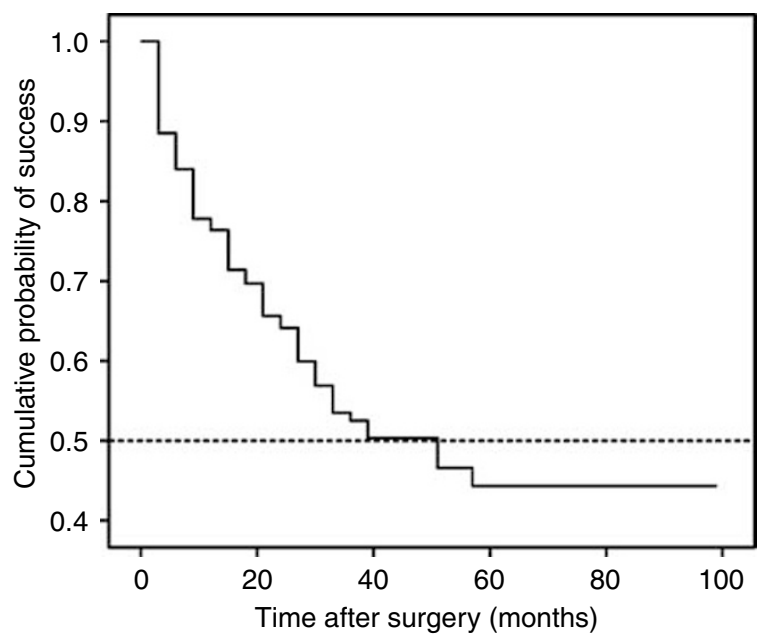

Figure 1 Survival curves as a function of time after surgery. Survival analysis showed that recurrence rates increased with time and that the median recurrence time from surgery to deviation recurrence was 48.3 months.

$>10$ PD of esotropia/phoria, 4.8-fold higher for those with 0-10 PD of esotropia/phoria, and 1.9-fold higher for those with 1-10 PD of exotropia/phoria. 
Table 2 Deviation at postoperative day one in the successful and recurrence groups

\begin{tabular}{lccr}
\hline \begin{tabular}{l} 
Alignment at $\begin{array}{l}\text { Nostoperative day 1 } \\
\text { postal }\end{array}$ \\
\cline { 2 - 3 }
\end{tabular} & \multicolumn{2}{c}{ No. of patients } & \\
\cline { 2 - 3 }$>$ Recurrence (\%) & Success (\%) & \\
\hline 10 PD of esotropia/phoria & $28(19.7)$ & $114(80.3)$ & 142 \\
$1-10$ PD of esotropia/phoria & $62(37.3)$ & $104(62.7)$ & 166 \\
$>10$ PD of exotropia/phoria & $30(66.7)$ & $15(33.3)$ & 45 \\
Total & $9(75.0)$ & $3(25.0)$ & 12 \\
\hline$P$ & 129 & 236 & 365 \\
\hline
\end{tabular}

$P<0.001$

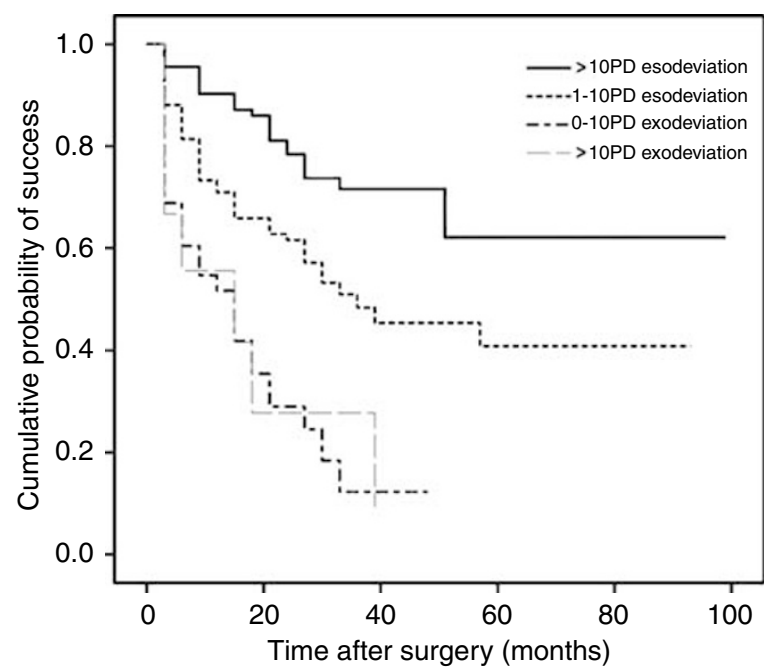

Figure 2 Survival curves for each of the four postoperative alignment groups. Initial postoperative alignment significantly affected outcome in exotropia surgery. The survival curve indicated that the median recurrence time was 99.0 months in patients with an initial alignment of $>10 \mathrm{PD}$ of esotropia/ phoria, 34.1 months in those with 0-10 PD of esotropia/phoria, and 13.2 months in those with 1-10 PD of exotropia/phoria.

\section{Discussion}

Of the patients after exotropia surgery, $60.3 \%$ had a successful outcome. This result is comparable to those of other authors, ${ }^{1-6}$ although the criteria associated with a successful outcome and the length of follow-up vary widely in reports.

In this study, early postoperative deviation was the only factor found to determine the outcome of exotropia surgery. Other authors have identified preoperative factors such as age at surgery; size, comitance, or constancy of deviation; presence of amblyopia or anisometropia; or degree of sensory binocularity as important for long-term success. ${ }^{1-8}$ We found none of these to be important in the survival analysis. Additionally, vertical transposition of horizontal rectus muscles or surgery of vertical rectus muscles or oblique muscle, in combination with bilateral rectus recession, did not significantly affect outcome.

We used survival analysis to investigate the factors affecting outcome. This is a particularly useful method for assessing the importance of covariates in situations where patients have been followed up for various periods. ${ }^{4}$ Moreover, Cox multivariate survival analysis provides both survival analysis and stepwise forward multiple regression analysis. In the survival analysis, patients who had not become failures were allowed to contribute information irrespective of the follow-up time. This method provides estimates of expected failure times based on each particular patient's covariates, and allows predictions to be made concerning the effect of each particular covariate. For our patients, the overall estimated median time to failure was 48.3 months. Since early postoperative alignment was a predictive covariate in our study, patients with an initial alignment of $>10$ PD of esotropia/phoria had an estimated median recurrence time of 99.0 months, while those with $0-10$ PD of esotropia/phoria had an estimated median recurrence time of 34.1 months.

Postoperative large angle esotropia, being desirable for long-term prevention of recurrence, may cause a monofixation syndrome with loss of stereopsis and increase the risk of needing to intervene for a nonresolving esotropia. However, in another study we previously reported, ${ }^{15}$ we found that an initial overcorrection of $20 \mathrm{PD}$ or more after surgery for exodeviation was reduced to 10 PD or less at distance and at near within a postoperative 4 weeks in most patients and that reoperation for consecutive esotropia was necessary in only $5.9 \%$ of the patients.

In conclusion, we found early postoperative overcorrection to be the only predictor of a successful long-term outcome after exotropia surgery. Purposeful overcorrection of the exodeviation tended to ensure a more satisfactory result.

\section{References}

1 Clarke WN, Noel LP. Surgical results in intermittent exotropia. Can J Ophthalmol 1981; 16: 66-69.

2 Scott WE, Keech R, Mash AJ. The postoperative results and stability of exodeviations. Arch Ophthalmol 1981; 99: 1814-1818.

3 Beneish R, Flanders M. The role of stereopsis and early postoperative alignment in long-term results of intermittent exotropia. Can J Ophthalmol 1994; 29: 119-124.

4 Stoller SH, Simon JW, Lininger LL. Bilateral lateral rectus recession for exotropia: a survival analysis. J Pediatr Ophthalmol Strabismus 1994; 31: 89-92.

5 Richard JM, Parks MM. Intermittent exotropia: surgical results in different age groups. Ophthalmology 1983; 90: 1172-1177. 
6 Pratt-Johnson JA, Barlow JM, Tillson G. Early surgery in intermittent exotropia. Am J Ophthalmol 1977; 84: 689-694.

7 Gezer A, Sezen F, Nasri N, Gozum N. Factors influencing the outcome of strabismus surgery in patients with exotropia. J Pediatr Ophthalmol Strabismus 2004; 8: 56-60.

8 Abbasoglu OE, Sener EC, Sanac AS. Factors influencing the successful outcome and response in strabismus surgery. Eye 1996; 10: 315-320.

9 McNeer KW. Observations on the surgical overcorrection of childhood intermittent exotropia. Am Orthopt J 1987; 37: 135-150.

10 Ruttum MS. Initial versus subsequent postoperative motor alignment in intermittent exotropia. J Pediatr Ophthalmol Strabismus 1997; 1: 88-91.
11 Freeman RS, Isenberg SJ. The use of part-time occlusion for early onset unilateral exotropia. J Pediatr Ophthalmol Strabismus 1989; 26: 94-96.

12 Wright KW. Strategies and techniques. In: Wright KW (eds). Color Atlas of Strabismus Surgery, 2nd ed. Wright Publishing: Republic of Panama, 2000, pp 249-250.

13 Kim C, Hwang JM. Largest angle to target in surgery for intermittent exotropia. Eye 2005; 19: 637-642.

14 Burian HM. Exodeviations: their classification, diagnosis, and treatment. Am J Ophthalmol 1966; 62: 1161-1166.

15 Kim T, Kim JH, Hwang JM. Long term outcome of patients with large overcorrection following surgery for exotropia. Ophthalmologica 2005; 219: 237-242. 